\title{
There Is No Measurement Problem for Humeans*
}

\author{
Chris Dorst \\ Forthcoming in Nô̂s. (Please cite published version.)
}

\begin{abstract}
The measurement problem concerns an apparent conflict between the two fundamental principles of quantum mechanics, namely the Schrödinger equation and the measurement postulate. These principles describe inconsistent behavior for quantum systems in so-called "measurement contexts." Many theorists have thought that the measurement problem can only be resolved by proposing a mechanistic explanation of (genuine or apparent) wavefunction collapse that avoids explicit reference to "measurement." However, I argue here that the measurement problem dissolves if we accept Humeanism about laws of nature. On a Humean metaphysics, there is no conflict between the two principles, nor is there any inherent problem with the concept of "measurement" figuring into the account of collapse.
\end{abstract}

\section{Introduction}

Standard presentations of quantum mechanics posit two fundamental principles. The Schrödinger equation describes how the state of a quantum system evolves over time when it is not being measured. The measurement postulate describes how a system evolves when it is being measured, and provides the probabilities that, upon measurement, the system will be found to have a certain value (or a value within a certain range) of some dynamical variable. These principles are quite different - for example, the former is deterministic, while the latter is stochastic - and so it does not look like either can be derived from the other.

This situation has struck some physicists and many philosophers as problematic, and has come to be known as the measurement problem. At first glance, the proffered reasons for the problem are not always the same. Some authors claim that the measurement postulate, with its application restricted to "measurement contexts," is unacceptably imprecise. ${ }^{1}$ Others worry that there is no characterization of "measurement" in quantum mechanical terms. ${ }^{2}$ And still

\footnotetext{
*Thanks to Eddy Keming Chen, Stanley Dorst, Ned Hall, Michael Hicks, and Robert Smithson for very helpful comments and discussion. Also thanks to several anonymous referees for their probing criticisms and insightful questions.

${ }^{1}$ Bell (1990, p. 33); Lewis (2016, p. 49).

${ }^{2}$ Ismael (2000); Hughes (1989, p. 278).
} 
others find this "strange dualism" in the fundamental quantum principles to be inherently inconsistent. ${ }^{3}$ But beyond these more refined objections, at the very least it ought to strike us as somewhat strange that nature seems to act one way when it is being measured, and another way when it is not being measured.

However, there is a way of understanding all of these objections as motivated by the same core idea. My goal here is to argue that once we articulate that idea clearly, we will see that it presupposes a specific conception of the metaphysics of laws of nature. In particular, the measurement problem tacitly assumes an anti-Humean metaphysics of laws, according to which the laws are principles that govern the behavior of physical systems. If we instead adopt a Humean view of laws, whereby the laws are some sort of description of the behavior of physical systems, then the measurement problem no longer arises. To put it concretely, I will argue that the Humean can take seriously the possibility that the Schrödinger equation and the measurement postulate together comprise the fundamental quantum mechanical laws of nature. ${ }^{4}$

Before proceeding, however, it's worth pausing a moment to consider why this matters. After all, we already have theories that purport to solve the measurement problem - indeed, that is one of the primary tasks of various interpretations of quantum mechanics such as Many Worlds, Bohmian Mechanics, and GRW. If these theories have already solved the problem, why does it matter that Humeanism dissolves it?

It matters for at least two reasons. First, because it is not quite correct to say that these theories solve the measurement problem. Rather, they would solve it if they were true. But of course, none of these theories is without its characteristic problems. For example, Many Worlds has well-known problems understanding quantum probabilities; Bohmian Mechanics struggles to explain how a wavefunction defined on configuration space can guide particles located in familiar three-dimensional space; and GRW has enough issues that I can't pick just one to highlight. ${ }^{5}$

So it's worth considering whether there are other options. Must one of these interpretations, or perhaps a close cousin, provide the correct solution to the measurement problem? I'll argue that the answer is negative: accepting Humeanism shows that the search for a mechanism of (genuine or apparent) wavefunction collapse might have been the search for a chimera. (That said, I don't mean to imply that there is no need for a Humean interpretation of quantum mechanics. I'll have more to say about the connection between the Humean dissolution and the question of interpretation in $\S 6$.)

The second reason it matters that Humeanism dissolves the measurement problem is because it provides evidence for Humeanism. Historically speaking,

\footnotetext{
${ }^{3}$ Albert (1992, pp. 78-79); Lewis (2016, p. 50). The phrase "strange dualism" is due to Wigner (1963, p. 7).

${ }^{4} \mathrm{I}$ will not argue that these two principles definitively are the fundamental laws, on the Humean view, only that there is a reasonable case to be made that they are.

${ }^{5}$ Among them are the "tails problem" (Lewis, 1995), the requirement that systems do not technically collapse onto a position eigenstate (but only close to a position eigenstate), and the issue that not all conceivable measurement outcomes are recorded in macroscopic positions (Albert, 1992).
} 
the widespread adoption of the Copenhagen interpretation in the early twentieth century looks rather mysterious if it was plainly evident from the start that, because of the measurement problem, its proposal for the quantum mechanical laws simply could not have been correct. Now, one might take this to be evidence that physicists treated the Copenhagen interpretation much like they now treat Newtonian mechanics: as a useful but false approximation. This is certainly possible, but it ignores a crucial difference between the two. Newtonian mechanics was at least possibly correct, and was largely believed to be so when it was first adopted. By contrast, if the measurement problem is as troublesome as many theorists would have us believe, then the Copenhagen interpretation simply could not have been correct - there was no possible world with its laws of nature - and this should have been evident from the beginning. ${ }^{6}$

Why, then, did it become widely adopted? One possibility is that physicists are really instrumentalists. Readers of Bohr can attest to the plausibility of this suggestion at least in his case. We would then have to ask: If physicists are instrumentalists, what does that imply about the philosophical interpretation of physics? Should philosophers of physics also be instrumentalists?

Without trying to answer these questions, let me suggest another possibility: that many physicists really did think that the Copenhagen interpretation was correct. Its widespread adoption then wouldn't have been due to a widespread instrumentalism among physicists, nor would it require us to view the majority of physicists as realists who knowingly adopted a false theory.

To make sense of this, we would need a realist view according to which the laws of the Copenhagen interpretation might have been correct. My suggestion is that Humeanism provides us with just such a view. If this is right, it shows that Humeanism is uniquely compatible with a realist interpretation of actual physical practice in the twentieth century. That, I take it, constitutes significant evidence for Humeanism over its alternatives. ${ }^{7}$

So much for the motivations. Let me outline where this paper is going. In §2 I review various statements of the measurement problem and articulate the core idea that I think motivates all of them. In $\S 3$, I review some recent developments in the Humean understanding of laws of nature, and in $\S 4$ I use these developments to argue that Humeanism effectively dissolves the measurement problem. In $\S 5$, I address an objection to this line of argument, namely that it is inconsistent with physical practice because it overgeneralizes, engendering an unwanted multiplicity of imprecise fundamental laws. In $\S 6$ I explore how the Humean dissolution of the measurement problem bears on the project of interpreting quantum mechanics. I conclude in $\S 7 .^{8}$

\footnotetext{
${ }^{6} \mathrm{I}$ am relying here on the claim that the Schrödinger equation and the measurement postulate (the two fundamental laws of the Copenhagen interpretation) are inconsistent. If that's right, then since no world can have inconsistent laws, no world can have the laws of the Copenhagen interpretation. See $\S 2$, particularly the third conception of the measurement problem therein, for further discussion.

${ }^{7}$ To be clear, I am not suggesting that early twentieth-century physicists were explicitly Humean. I am suggesting that insofar as philosophers want to be able to provide a realist interpretation of their practices, we are pressed to adopt Humeanism.

${ }^{8}$ One more clarification before proceeding: I restrict myself here to issues that are "internal"
} 


\section{What Exactly is the Measurement Problem?}

To understand the measurement problem, it is important to appreciate that the Schrödinger equation and the measurement postulate are significantly different principles. Quantum mechanics represents the state of a system as a vector in a vector space, and the role of the Schrödinger equation is to describe how that state vector evolves over time. It has the following form:

$$
i \hbar \frac{\partial}{\partial t}|\psi\rangle=\hat{H}|\psi\rangle
$$

where $|\psi\rangle$ is the state vector of the quantum system in question and $\hat{H}$ is the system's Hamiltonian (a representation of the system's total energy that encodes its fundamental interactions). Significantly, this equation is linear, which means that it preserves superpositions.

The measurement postulate has a radically different form. When a system in state $|\psi\rangle$ undergoes a measurement of dynamical variable $A$, the measurement postulate gives us the probability of that measurement obtaining value $a_{i}$ using the Born rule:

$$
\operatorname{Pr}_{\psi}\left(A=a_{i}\right)=\left\langle\psi\left|\hat{P}^{A}\left(a_{i}\right)\right| \psi\right\rangle
$$

where $\hat{P}^{A}\left(a_{i}\right)$ is a projection operator onto the subspace in the vector space associated with the dynamical variable $A$ having value $a_{i}$. Moreover, the measurement postulate adds the additional claim that when the value $a_{i}$ is obtained, the state of the system "collapses" from $|\psi\rangle$ to $\hat{P}^{A}\left(a_{i}\right)|\psi\rangle$. These collapses do not preserve superpositions, and they never occur as a result of the operation of the Schrödinger dynamics alone.

In summary, then, the difference in the principles are twofold: first, the Schrödinger dynamics is deterministic whereas the measurement postulate is stochastic, and second, the measurement postulate posits a distinct physical collapse process that has no place in the Schrödinger dynamics. These differences make it seem unlikely that either principle could be derived as a special case of the other. ${ }^{9}$

It may not be obvious why this radical difference between the two basic quantum mechanical principles is problematic, and indeed different authors have advanced different worries about it. One of the most vigorous presentations of the seriousness of the problem is found in John Stuart Bell's (1990) paper, "Against 'Measurement,"' which begins:

Surely, after 62 years, we should have an exact formulation of some serious part of quantum mechanics? By 'exact' I do not of course

to quantum mechanics. There are plenty of worries, of course, about what happens when we try to combine quantum mechanics with general relativity, but I will not be concerned with those issues in this paper.

${ }^{9}$ There are some recent attempts to derive the measurement postulate from the Schrödinger equation, at least in specific measurement contexts; see, for example, Allahverdyan et. al. (2013, 2017) and Deumens (2019). The success of these derivations is, at this point, still an open question. Vaidman (2020) provides a critical evaluation of these proposals. 
mean 'exactly true'. I mean only that the theory should be fully formulated in mathematical terms, with nothing left to the discretion of the theoretical physicist... (p. 33)

Bell's concern here is with the measurement postulate, and the idea that it is to be applied in "measurement contexts." As Bell shows, the question of exactly what counts as a measurement context is left unanswered in standard textbook treatments of quantum mechanics, thus leaving it up to the physicist to determine when to apply the Born rule. ${ }^{10}$

Now, a common response to this worry is that the question of precisely when a measurement occurs does not need to be answered: in learning quantum mechanics, physicists gain an intuitive understanding of which situations count as measurement contexts and which do not, so in actual applications of the theory there is no problem. ${ }^{11}$ But Bell makes clear that the problem is not a practical one. Indeed, he coins the acronym FAPP, "for all practical purposes," and allows that standard formulations of quantum mechanics are perfectly fine FAPP. His complaint, rather, is that a term like "measurement" should not appear in a fundamental physical theory simply because it is inexact. A better theory, he thinks, would give us a more precise characterization of exactly when the measurement postulate applies.

Peter Lewis (2016, pp. 49-50) makes the same point more starkly. Consider Schrödinger's cat, isolated in a box with a vial of poison that will be released if and only if a radioactive atom decays within an hour. By the Schrödinger equation, the radioactive atom will evolve into a superposition of both decayed and not decayed states. This also implies that the vial of poison will evolve into a superposition of released and unreleased, and the cat will evolve into a superposition of alive and dead. Eventually, we open the box and observe the contents, finding either an alive cat with an unbroken vial and undecayed atom, or a dead cat with a broken vial and decayed atom. We do not find a system in superposition, because at some point in this process a measurement of the state of the system occurred, collapsing it one way or the other. But at which point did the measurement occur? When the decay was detected? Or when the cat heard the vial break? Or when the cat died? Or when we opened the box? Without a more precise characterization of "measurement," quantum mechanics does not answer this question, and this imprecision is what Bell thinks is unacceptable. ${ }^{12}$

\footnotetext{
${ }^{10}$ Bell's example texts include Dirac (1948), Gottfried (1966), and Landau and Lifschitz (1977).

${ }^{11}$ Compare, e.g., Maudlin (2019), pp. 3-4: "The imprecisions in the formulation of the quantum mechanical recipe do not have noticeable practical effects when it comes to making predictions. Physicists know well enough when a certain laboratory operation is to count as an observation, and what it is an observation of." Maudlin here speaks of "observations," though I prefer the somewhat-more-standard talk of "measurements" because it is less committal about the presence of an agent.

${ }^{12}$ This relatively standard presentation is embellished somewhat. Most physicists would probably agree that a measurement of the state of the atom has occurred by the time the decay is detected and the mechanism for breaking the vial has initiated. See e.g. Bohr and Kalckar (1985, pp. 451-454) and Carpenter and Anderson (2006) for further discussion.
} 
Other statements of the measurement problem focus on a related point: that we have no characterization of "measurement" in quantum mechanical terms. Rather, we have only the intuitive grasp that physicists gain, in the process of learning quantum mechanics, about when a measurement has occurred. Ismael (2000), for example, notes that "nobody has managed to say in a completely satisfactory way, in the terms provided by the theory, which contexts are measurement contexts." Similarly, R. I. G. Hughes says that the measurement problem requires us to explicate "what it is that physically distinguishes the kinds of interactions governed by Schrödinger's equation from those in which discontinuous changes (allegedly) occur" (1989, p. 278).

Note that these statements of the problem are at least superficially distinct from Bell's. The objection here is that there is something inherently unsatisfactory about the fact that we have no quantum-mechanical explication of "measurement contexts." That is taken, in and of itself, to be a problem. Bell, on the other hand, is concerned with the imprecision that results from this lack of a quantum-mechanical explication. To see the difference, note that if we somehow had a precise characterization, in non-quantum-mechanical terms, of when the collapse occurs, then Bell's worry would be addressed, but the present one would not.

One might wonder why it is problematic that we lack a quantum-mechanical account of measurement. The answer lies in a third way of articulating the measurement problem: finding an inconsistency in the fundamental principles. Lewis again provides an apt summary of this worry:

What is a measurement? At bottom, it is a physical process like any other: Measuring devices are just hunks of matter, obeying the same physical laws as any other hunk of matter. But then it is simply impossible for the physical laws to differ between measurements and nonmeasurements, as the measurement postulate requires... Measuring devices must obey the Schrödinger dynamics, since every physical system does, but they must also violate the Schrödinger dynamics if they are to enact the measurement postulate. Quantum mechanics, understood as including the measurement postulate, is not just incomplete; it is inconsistent" (2016, p. 50). ${ }^{13}$

The idea here seems straightforward at first glance, but it has its subtleties. To see it more clearly, note that we may give a piecewise formulation of the laws of standard textbook quantum mechanics describing the dynamical evolution of a given system as follows:

$$
\left\{\begin{array}{l}
\text { Schrödinger equation } \quad \text { if system is not measured } \\
\text { Measurement postulate } \\
\text { (Born rule }+ \text { collapse } \\
\text { onto eigenstate of } A \text { ) }
\end{array}\right.
$$

${ }^{13}$ Albert (1992, pp. 73-79) finds a similar contradiction between the two principles. 
There is no formal inconsistency here, so one might well wonder what Lewis's objection is. The worry (to put it somewhat figuratively) is that nature can only understand conditions that are characterized in the language of fundamental physics. For example, it can understand the behaviors described on the left side, but it cannot understand the conditions described on the right, because those conditions make reference to measurement, which is not in the language of fundamental physics. So nature cannot be "looking out" for measurements as a way of knowing when to deviate from the Schrödinger equation. Hence systems will always obey the Schrödinger equation, and will thus behave inconsistently with the measurement postulate in measurement contexts.

This leads us to the core idea behind all of these statements of the measurement problem. It is not enough that we can distinguish when to apply either the Schrödinger equation or the measurement postulate. Rather, nature itself must somehow be able to distinguish which sort of context it's in. But on what basis does it do so? Since it doesn't pay attention to "measurements" (at least not qua measurements), our piecewise statement of the laws that does appeal to measurements cannot be correct. This is why it is problematic that we lack a quantum mechanical account of measurement, and also why people like Lewis and Albert have discerned an inconsistency in the fundamental principles. The inconsistency rests on the assumption that these principles are, essentially, the rules by which nature governs the world. If that assumption is right, then nature must be able to "tell" which rule applies in which conditions. And if that assumption is right, then however nature does tell which rule applies, the conditions that it uses must be perfectly precise (articulated in the language of fundamental physics) and leave no room for ambiguity, thus addressing Bell's worry about imprecision. But is that assumption right? ${ }^{14}$

\footnotetext{
${ }^{14}$ Another way of presenting the measurement problem, distinct from those canvassed above, can be found in Maudlin (1995, p. 7). According to Maudlin, the problem is that no theory can accept all three of the following claims: (1) a system's wavefunction specifies all of its physical properties, (2) the wavefunction always evolves in accord with the Schrödinger equation, and (3) measurements have definite outcomes.

I do not think that there is anything wrong with Maudlin's construal, but note that it locates the measurement problem prior to the measurement postulate, which itself constitutes a rejection of claim (2). Standard textbook presentations of quantum mechanics, replete with the measurement postulate, would therefore count as a solution to the measurement problem on Maudlin's construal. Nevertheless, many people - including the authors cited above, and indeed including Maudlin himself (ibid., p. 9) - take the standard textbook presentation to be inadequate precisely because it includes the measurement postulate. It therefore seems more natural to treat the measurement problem as an issue with the standard textbook presentation, rather than one that is resolved by that presentation.

Nothing much hinges on this, however. Adopting Maudlin's construal wouldn't change my substantive claims here; it would just necessitate a slight reframing. If, following Maudlin, we regard the standard textbook presentation of quantum mechanics as a solution to the measurement problem, then my claim is that whereas many theorists have thought it to be an unacceptable solution, Humeans should view it as unproblematic.
} 


\section{Humeanism and Property Opportunism}

Humeanism about laws of nature is the view that laws are certain sorts of regularities in the totality of the particular matters of fact that obtain in the history of the universe. Nowadays the orthodox Humean view of laws of nature is David Lewis's Best System Account ("BSA"). ${ }^{15}$ According to the BSA, the laws of nature are the regularities of the simplest and strongest systematization of the "Humean mosaic."

Lewis had some specific ideas about the character of the particular matters of fact comprising the mosaic. He thought that they consist of spacetime points instantiating properties that are local, intrinsic, and non-modal. Exactly which properties get instantiated is a matter for fundamental physics to discover, but paradigmatic examples might be things like mass, charge, and spin. Lewis referred to the fundamental properties discovered by physics as "perfectly natural" properties to distinguish them from other properties that are metaphysically non-elite. The metaphysically non-elite properties include higher-level properties like being a brother, as well as gerrymandered or disjunctive properties like grue and its cognates. So the job of a Lewisian best system is to systematize the totality of facts about spacetime points instantiating perfectly natural properties.

As Loewer (1996) points out, Lewis's commitment to a semi-classical spacetime arena with localizable point properties is largely inessential to the broader picture provided by the BSA. For example, one could be a Humean about laws and think that the fundamental space is something like the configuration space on which the universal wavefunction is defined. ${ }^{16}$ What is important for Humeanism is that there are no necessary connections between the properties instantiated at different points in the fundamental space of the mosaic.

Lewisian systems take certain claims as axioms, which then deductively entail facts about the Humean mosaic. The simplicity of a system is a function of the axioms: how many there are, and how syntactically complex they are. Other things being equal, a simpler system is better. On the other hand, the strength of a system is a function of the amount of information it provides us about the particular matters of fact. Other things being equal, a stronger system is better.

The standards of simplicity and strength conflict with each other, so in systematizing the mosaic we look to find the best balance of the two. Why do we care about doing this? Here is David Albert explicating the rationale for seeking this balance by imagining a one-on-one conversation with God:

$[Y]$ ou ask if maybe there's something meaty and pithy and helpful

\footnotetext{
${ }^{15}$ See Lewis $(1973,1986,1994)$.

${ }^{16}$ It is less obvious to me whether one might consistently be both a Humean and a "Hilbert Space Fundamentalist," i.e. think that the fundamental space is a Hilbert space inhabited by the universal state vector (Carroll, forthcoming). What exactly does the Humean denial of necessary connections between distinct points in that space amount to? Certainly we can have the absence of necessary temporal connections here, but the denial of necessary spatial connections is harder to understand, since the entire universe is just a single vector evolving in the space.
} 
and informative and short that He might be able to tell you about the world which (you understand) would not amount to everything, or nearly everything, but would nonetheless still somehow amount to a lot. Something that will serve you well, or reasonably well, or as well as possible, in making your way about in the world. And what it is to be a law, and all it is to be a law, on this picture... is to be an element of the best possible answer to precisely this request. (2015, p. 23). ${ }^{17}$

This passage articulates the guiding idea behind the BSA: no matter what the fundamental ontology of the world is like - be it a collection of point particles moving around in a three-dimensional spacetime or a universal wavefunction evolving in configuration space - the laws of nature are concise summaries of the behavior of those entities that are useful in our attempts to navigate the world. The bumper sticker slogan for Humeanism about laws would be something like this: "The world acts; the laws describe." This slogan will be useful to keep in mind in what follows. ${ }^{18}$

One further element of the BSA will be crucial to the Humean dissolution of the measurement problem: a maneuver I call "property opportunism." Property opportunism is motivated by the pragmatic guiding idea, embodied in Albert's quote above, that laws of nature are meant to help us effectively navigate the universe. The maneuver has been developed and defended by Hicks and Schaffer (2017), and it concerns the kinds of properties that may figure into fundamental laws of nature. ${ }^{19}$

Traditionally, philosophers have thought that fundamental laws of nature may only appeal to fundamental properties. Lewis, for example, explicitly requires that laws of nature be formulated only in terms of perfectly natural properties (see, e.g., Lewis [1986, p. 368]). Similarly, Armstrong suggests that fundamental laws are nomic necessitation relations between universals, and universals constitute the metaphysically elite properties (see [Armstrong 1978, 1983]). And North (2013, p. 186) treats the idea that fundamental laws relate fundamental properties as essentially a methodological principle that guides our physical theorizing.

According to Hicks and Schaffer, however, there is reason to question this assumption, both from the perspective of Humeanism, and from the perspective of scientific practice. From the latter perspective, consider an equation like $F=m a$. In Newtonian mechanics, this equation is usually taken to express a fundamental law of nature. Within the classical presentation of that theory, however, Hicks and Schaffer argue that both $F$ and $a$ represent derivative, non-fundamental quantities. More specifically, a represents acceleration,

\footnotetext{
${ }^{17}$ See Lange (2009, pp. 101-102) and Beebee (2000, p. 574) for similar expressions of the same idea.

${ }^{18}$ It will turn out that there is actually an important ambiguity in this passage of Albert's, so we will return to it in $\S 5$. But for now, the basic guiding idea of laws as efficient summaries will be sufficient.

${ }^{19}$ Similar ideas are considered in Hall (ms), Bhogal and Perry (2017), and Esfeld (2020), among others.
} 
and in classical Newtonian mechanics, acceleration is defined as the second time derivative of absolute position: $a={ }_{d f} d^{2} x / d t^{2}$. Moreover, this definition is irreversible: one can derive accelerations from positions (over time), but one cannot recover positions from accelerations. The irreversibility of the definition of acceleration thus leads Hicks and Schaffer to conclude that acceleration is a derivative quantity in Newtonian mechanics, yet it appears in a fundamental law. ${ }^{20}$

Similarly, $F$ here stands for the resultant force on the object in question. Resultant force is defined as the vector sum of the component forces acting on the object. As with acceleration, this definition is irreversible: it is not possible to recover the component forces if all one knows is the resultant force. Hicks and Schaffer take this to indicate that resultant force is a derivative quantity in Newtonian mechanics, yet it appears in a fundamental law. So, scientific practice underwrites the permissibility of derivative properties in fundamental laws.

What motivation is there to include derivative properties in statements of fundamental laws? Hicks and Schaffer suggest three reasons: simplicity, transparency, and modularity. Simplicity is rather obvious: it is simpler to express these equations using the derivative notions of acceleration and resultant force than it is using the fundamental notions of position and component forces. One need only compare $F=m a$ with $\Sigma_{i=1}^{n} \vec{f}_{i}=m d^{2} x / d t^{2}$ to see this. As for transparency, Hicks and Schaffer maintain that using " $a$ " instead of " $d^{2} x / d t^{2}$ " is beneficial because it draws our attention to the variable that is dynamically relevant: accelerations, not absolute positions. Lastly, using $F$ instead of an expression of the component forces is helpful because it allows us to be neutral about which component forces there are: sometimes all one knows is the resultant force, and in such situations it would be problematic if one were required to write out the various unknown component forces instead.

One point to observe here is that Hicks and Schaffer are expanding the sorts of standards that get balanced in the candidate ranking of systematizations. In addition to simplicity and strength, we now have the additional standards of transparency and modularity, which we might group together under the heading of "tractability." On the view suggested here, the best system is the one that achieves the best balance of simplicity, strength, and tractability. ${ }^{21}$

So the motivations for allowing derivative properties in fundamental laws are explicitly pragmatic. As Hicks and Schaffer put it:

$[\mathrm{P}]$ hysicists are in practice happy to trade some metaphysical noneliteness for sufficient practical gains in matters such as mathemat-

\footnotetext{
${ }^{20}$ Granted, one could situate Newtonian mechanics in a Galilean spacetime that does not regard absolute spatial location as a well-defined quantity. Nevertheless, as Hicks and Schaffer point out, physicists did not await the discovery of such alternative spacetimes before regarding $F=m a$ as a fundamental law. This shows that physicists are willing to countenance nonfundamental quantities in fundamental laws.

${ }^{21}$ The suggestion to include "tractability" in the criteria for best system is made explicitly by Loewer (2020); other authors such as Jaag and Loew (2020) and Callender (2017, p. 162) have advocated similar ideas.
} 
ical simplicity and dynamical transparency. To the extent that we can expect the physicists to deliver certain basic equations at all, we can expect that their choice of equations will be partly guided by pragmatic constraints. (ibid., p. 428)

I call this view "property opportunism," because the idea is that laws are not constrained to reference only fundamental properties; rather, they can reference derivative properties if doing so makes them more useful in certain respects. ${ }^{22}$

So property opportunism seems consistent with scientific practice. It also accords well with the pragmatic motivations behind Humeanism. One way to see this is to state these examples in best-system terminology: the gain in simplicity and tractability achieved by using " $a$ " or " $F$ " rather than " $d^{2} x / d t^{2}$ " or " $\sum_{i=1}^{n} \overrightarrow{f_{i}}$ " could end up outweighing the minor loss in strength that accompanies such replacements. At the very least, we cannot conclude on a priori grounds that the benefits to simplicity and tractability of including derivative properties in fundamental laws will always be outweighed by any costs to strength.

By contrast, it is difficult to see how property opportunism could be accommodated on an anti-Humean view whereby laws are seen as metaphysically robust relations inhering in the world. If, as Armstrong would have it, laws are relations between universals, and universals correspond to the metaphysically elite properties, then there is no room for laws that have derivative properties as relata. Alternatively, if laws are fundamental elements of reality that produce subsequent states of the universe from its current state (as Maudlin (2007) maintains), it is hard to see how they could concern derivative properties without thereby engendering some strange sort of downward causation between the higher-level derivative properties and the lower-level fundamental properties. In light of these difficulties, property opportunism seems uniquely well-motivated from the Humean perspective.

Though property opportunism is usually regarded as a recent development in Humeanism, there is actually a sense in which Lewis himself was already committed to it once he offered his treatment of chance. Remember, chance is not a perfectly natural property, for Lewis. Nevertheless he allows that the fundamental laws might reference it, provided that by doing so they generate a better summary of the mosaic. So Lewis was already onboard with the idea that metaphysically derivative properties can figure in fundamental laws. Hicks and Schaffer are simply suggesting that this maneuver should be applied more liberally.

However, if we apply it too liberally, we may encounter problems. My concern here traces to Lewis's original motivation for requiring that laws reference only perfectly natural properties. Lewis was worried that the balance of simplic-

\footnotetext{
${ }^{22}$ Several other developments of the BSA are similarly friendly to property opportunism, such as Loewer's (2007, 2020) package deal account and Cohen and Callender's (2009) better best system account. Both of these accounts are flexible about the properties they allow to figure in the best system. Chen (forthcoming b) also explores the possibility that fundamental laws may employ vague predicates, where one source of vagueness may be that they refer to derivative, non-fundamental properties, such as "low entropy" (in statements of the Past Hypothesis).
} 
ity and strength could be trivialized if there were no constraints on what kinds of properties can figure into the laws. For example, if we take the property $F$ to apply to all and only the entities that exist in this universe, then one could achieve maximal simplicity and strength just by using the expression $\forall x F x$. But presumably we don't want $\forall x F x$ to count as a law of nature; it doesn't seem very lawlike. Lewis's solution was to require that laws reference only perfectly natural properties, and by Lewis's lights, $F$ doesn't count as perfectly natural.

Of course, this wasn't really a solution at all, given Lewis's treatment of chance as a metaphysically derivative (non-perfectly-natural) property. Saying that laws can only reference perfectly natural properties would either preclude Lewis from giving his treatment of chance, or it would commit him to the claim that fundamental laws cannot be chancy: indeterminism is metaphysically impossible. Presumably he wouldn't have been happy with either of these results. Nevertheless, we need some sort of restriction on what properties can figure in fundamental laws, lest we run headlong into Lewis's trivialization worry.

And property opportunism seems to be running in exactly that direction: it says that fundamental laws may reference derivative properties if the payoff is good enough. So what's to stop us from positing a property like $F$, and thereby the law $\forall x F x$, on this view? Isn't the payoff of doing so really tremendous?

Well, no. Admittedly, the payoff is significant in terms of simplicity and strength, but introducing $F$ into our systematization also engenders a massive decrease in tractability. One way to see this is to return to Albert's articulation of the pragmatic motivations behind the Humean picture using an imaginary conversation with God. Imagine that you ask God for some principles that "will serve you well, or reasonably well, or as well as possible, in making your way about in the world." And imagine that God replies, "Okay, I'm going to stipulate that an entity possesses the property $F$ if and only if it exists in precisely this universe. Here is your maximally useful principle: $\forall x F x$. Now, go forth."

If that is God's answer, then we would be justified in feeling a bit exasperated: $\forall x F x$ doesn't tell us anything that will help us make our way around in the world. We wanted something that was "meaty and pithy and helpful and informative" as Albert put it. But there is nothing that is genuinely meaty nor pithy nor helpful nor informative about God's principle here. It's a cheap syntactic trick, and its cheapness manifests in its uselessness.

The upshot is that if God is really going to answer our question helpfully, she will have to make sure that whatever principles she provides us make reference to properties that we can actually use for navigational purposes. This implies both that (i) the properties in God's principles must be cognitively graspable by creatures like us, and (ii) we must have some reasonable hope of being able to ascertain whether actual entities possess these properties.

In short, the basic pragmatic motivations behind Humeanism already generate tractability-related restrictions on the properties that can figure into the laws. These restrictions rule out Lewis's property $F$, but they do not rule out the possibility of using non-fundamental properties like Newtonian acceleration or resultant force - for those are things that we can get a cognitive grip on and know how to apply. So adopting property opportunism allows for more flexibil- 
ity in the kinds of properties that the laws can reference, but it does not invite the trivialization worry that Lewis was concerned about.

One final point needs addressing here. Once we allow that derivative properties may figure into fundamental laws, we face the question of how to draw the distinction between fundamental and derivative laws in the first place. For a natural proposal is that the fundamental laws are those laws that are stated perfectly precisely in the language of fundamental physics - but we cannot adopt this proposal if we are going to allow that fundamental laws may reference derivative properties.

Following Chen (forthcoming b, p. 10), I think the best response for a proponent of property opportunism is to adopt a necessary condition on derivative lawhood, as follows: if a law is derivative, then it can be derived (non-trivially) from the fundamental laws. Thus a law that cannot be so derived would be fundamental. Of course, all laws can be derived from themselves, but this would count as a trivial derivation. A non-trivial derivation would be one that requires additional assumptions or boundary conditions to be specified before the derivation can be completed. ${ }^{23}$ This necessary condition on derivative lawhood gets reasonable results. For example, various laws of chemistry and biology can plausibly be derived from fundamental physical laws once boundary conditions are specified and certain approximations are made.

\section{How Humeanism Dissolves the Measurement Problem}

We are now positioned to see how Humeanism dissolves the quantum measurement problem. The key move relies on property opportunism. Derivative properties may figure into fundamental laws if, by doing so, they make those laws more useful on the whole in our attempts to navigate the universe -i.e. if they engender a significant gain in either simplicity or tractability or strength at a minor cost to the other(s).

Consider then the property of being measured. That is, no doubt, a derivative property: it is not formulated in the language of fundamental physics, and it is not even clear how it could be so formulated. But it is hard to see why this should worry the Humean. In practice, as we saw, physicists are able to tell well enough whether a system is being measured or not. Indeed, Bell explicitly admits this in saying that traditional quantum mechanics with the measurement postulate is perfectly fine for all practical purposes. So from the Humean perspective, it should at least be an open question whether the best way of summarizing the mosaic makes reference to the property of being measured.

This move is suggestive. But to see more clearly why the measurement problem dissolves on the Humean picture, we need to consider each of the three statements of the problem that we saw in $\S 2$ : (1) "measurement" is imprecise, (2) we have no quantum mechanical account of measurement, and (3) the

\footnotetext{
${ }^{23}$ We do not claim that (non-trivial) derivability from fundamental laws is sufficient for derivative lawhood, lest we turn every fact about the mosaic into a law. Though it is an important project, I shall make no attempt to offer necessary and sufficient conditions for derivative lawhood in this paper.
} 
Schrödinger equation and the measurement postulate are inconsistent. If we accept Humeanism about laws, each of these worries loses its bite.

\subsection{Inconsistency and the Account of Measurement}

First consider worries (2) and (3). We saw that these worries hang together: the claim that the Schrödinger equation and the measurement postulate are inconsistent itself rests on the idea that if there is no quantum-mechanical account of measurement, then there will never be any reason for a quantum system to stop obeying the Schrödinger equation. So the two principles describe inconsistent behavior in measurement contexts.

When we explicated this problem in $\S 2$, the way we put it was that, absent a quantum mechanical account of measurement, nature has no way to tell when quantum systems should diverge from the Schrödinger dynamics because it doesn't speak the language of measurements. This talk of nature "being able to tell" was of course somewhat figurative, but nevertheless the Humean should view it as hopelessly misleading. Remember the Humean's bumper sticker slogan: The world acts; the laws describe. On this picture, nature does not need to tell when it is in a measurement context. It just behaves - unthinkingly - in manner A at some times, and in manner B at other times. Part of our task in identifying the laws is to find a simple, informative, and tractable way of distinguishing when it acts in which manner. And it may well turn out that the all-things-considered best way to do so is by appealing to the notion of measurement.

Certainly if we make the anti-Humean assumption that the laws are the rules by which nature governs the world, then it does not matter whether we can distinguish the conditions in which either the Schrödinger equation or the measurement postulate applies; it matters whether nature can do so. But from the Humean perspective, things are precisely reversed: what matters is whether we can effectively distinguish which principle applies in which cases, not whether nature can do so. The Humean thus rejects the fundamental assumption that generates the measurement problem in the first place. To put it in terms of our motivating idea, it is entirely possible that God would respond to our request for maximally useful navigational principles by providing us with the piecewise statement of the laws of textbook quantum mechanics that we saw in $\S 2$. It would be no objection to complain to God, "But how does nature know which principle she is to apply in which contexts?" That wasn't the game here; these were supposed to be navigational principles for us, not rules for nature.

\subsection{Imprecision}

Now consider worry (1): "measurement" is imprecise, and this imprecision means that we cannot always pinpoint precisely where the Schrödinger dynamics gives way to the measurement postulate. We saw this in the case of Schrödinger's cat, wherein quantum mechanics could not tell us precisely when the superposition collapsed. So adopting "measurement" as a characterization of when one 
principle gives way to the other does introduce a cost to the strength of our systematization. It might be suggested that this reduction in strength is too extreme to justify using the notion of "measurement" to mark the distinction between the conditions in which each principle applies.

But whether or not that is true will depend on what other methods there are for drawing that distinction. It may help to appreciate the efficiency of the traditional measurement formulation if we examine a particular alternative. Consider the following proposal for the fundamental quantum mechanical laws:

$$
\begin{cases}\text { Schrödinger dynamics } & \text { except at spatiotemporal locations } l_{17}, l_{58}, l_{829}, \ldots \\ \left|\psi_{1}\right\rangle \rightarrow \hat{P}^{A}\left(a_{i}\right)\left|\psi_{1}\right\rangle & \text { at } l_{17} \\ \left|\psi_{2}\right\rangle \rightarrow \hat{P}^{B}\left(b_{k}\right)\left|\psi_{2}\right\rangle & \text { at } l_{58} \\ \vdots & \vdots\end{cases}
$$

This formulation works by specifying a "default" dynamics (i.e. the Schrödinger equation) and then simply listing all the occasions on which systems will diverge from that dynamics. The subsequent lines tell us which wavefunctions collapse to which eigenstates of which variables at which spatiotemporal locations. Since it works by specifying the spatiotemporal locations at which collapses occur, we might call this candidate system the "Collapse Enumeration" systematization, $\mathcal{S}_{c e}$.

$\mathcal{S}_{c e}$ is arguably formulated in the language of perfectly natural properties (assuming spacetime points count as perfectly natural). Moreover, it is deterministic: it tells us not only where and when collapses occur, but also what state the system will collapse to. It has these features primarily because there is no plausible middle ground once we have decided to list all the divergences from the Schrödinger dynamics. In theory, we might instead just specify that the Schrödinger equation holds except at such-and-such spatiotemporal locations, and then simply not say what happens at those locations. Or we might say that wavefunctions collapse there but not specify which wavefunction does. Or we might specify which wavefunction collapses at each spacetime point but not specify what it collapses to. But once we've chosen to list each occurrence of collapse, it's hard to motivate omitting the further information about which wavefunction collapses and what it collapses to.

Plainly, $\mathcal{S}_{c e}$ would be both massively complex and hopelessly unwieldy. Even disregarding the fact that it's unclear how we could ever confirm such a theory, any time we wanted to predict how a quantum system was going to behave, we would have to consult this tedious list of spatiotemporal locations at which quantum systems diverge from the Schrödinger dynamics. By comparison, we perhaps ought to feel lucky that a much more compact and tractable formulation is available for distinguishing which principle applies at which times: textbook quantum mechanics with the traditional measurement postulate, the laws of which we stated in piecewise fashion back in $\S 2$. We might call this the "Measurement" systematization, $\mathcal{S}_{m}$. Granted, $\mathcal{S}_{m}$ appeals to the derivative and 
somewhat-imprecise property of being measured, but any associated costs in informativeness here should strike us as minor in comparison with the massive benefits in simplicity and tractability. Clearly, if the comparison is between $\mathcal{S}_{c e}$ and $\mathcal{S}_{m}$, and our question is which of these is more helpful in our attempts to navigate the universe, $\mathcal{S}_{m}$ is far superior. So the complaint that "measurement" is imprecise is by no means a decisive objection to $\mathcal{S}_{m}$.

Still, one might worry that the imprecision engendered by the measurement postulate is more insidious than I have suggested, because it prevents us from making predictions in certain situations where a more precise theory would not. In the Schrödinger's cat case, the imprecision concerns when, exactly, the collapse occurs within a certain interval. Nevertheless we can be certain that the measurement will have occurred by the time we open the box and examine its contents. So there is a sense in which the imprecision in this example is isolated: it's confined within the interval between the sealing and the opening of the box. It never "escapes the box" and ramifies outward to infect our predictions about what will happen in the rest of the universe.

But there are possible experiments that would, as it were, let the imprecision escape. Suppose, for example, we have a measuring device $\mathcal{M}_{1}$ set up inside the box to measure the cat's heartbeat to see if it's still alive. And suppose we have a second measuring device $\mathcal{M}_{2}$ set up to measure some complex, appropriatelyselected property of the composite [atom + vial + cat $\left.+\mathcal{M}_{1}\right]$ system. Even if we agree that $\mathcal{M}_{1}$ is a genuine measuring device and therefore will collapse the wavefunction (as per the measurement postulate), due to the vagueness of "measurement" there will be some imprecision concerning exactly when the $\mathcal{M}_{1}$ measurement has occurred. So $\mathcal{S}_{m}$ will not give us a precise point at which the wavefunction of the [atom + vial + cat $+\mathcal{M}_{1}$ ] system has collapsed; it will only give us an interval in which the collapse occurred.

Now suppose that $\mathcal{M}_{2}$ performs its measurement faster than $\mathcal{M}_{1}$, and it does so sometime within this interval. The statistical distribution of outcomes we would expect to get from $\mathcal{M}_{2}$ would then differ depending on whether the collapse of the $\left[\right.$ atom + vial + cat $\left.+\mathcal{M}_{1}\right]$ system has occurred by the time $\mathcal{M}_{2}$ makes its measurement. If the collapse has not already occurred, then for an appropriately-constructed $\mathcal{M}_{2}$ device, a single outcome is guaranteed. If the collapse has occurred, then we should expect a statistical distribution of different possible outcomes from $\mathcal{M}_{2} \cdot{ }^{24}$ The vagueness concerning exactly when the $\mathcal{M}_{1}$ measurement occurs (and thus, according to $\mathcal{S}_{m}$, when the wavefunction collapses) will then infect the outcome of the $\mathcal{M}_{2}$ measurement: $\mathcal{S}_{m}$ will make no definite predictions, not even probabilistic ones, about that measurement result. We might call this a nomological gap: a situation that the laws say is physically possible, but for which they license no predictions.

Adopting $\mathcal{S}_{m}$ thus implies that we have to countenance the possibility of nomological gaps. This has precedence in the work of Cartwright (see especially her (1999)), though she situates such gaps in an anti-Humean metaphysics, and we have already seen that the anti-Humean has independent reasons for

${ }^{24}$ See Albert (1992, pp. 84-92) for an extended discussion of an example of this sort. 
rejecting $\mathcal{S}_{m}$. The question for our purposes is whether the Humean can tolerate such a possibility, or if instead a system's countenancing nomological gaps automatically makes it unqualified to be the best system.

As far as I can see, nomological gaps are potentially worrisome, but they are not necessarily a dealbreaker for the Humean. As with other theoretical posits, they will be accounted for in the balance of simplicity, strength, and tractability. The main place they would seem to be problematic in this regard is with respect to strength: since the laws don't tell us what happens in these gaps, they are less informative than we might hope. But the amount of information we lose by positing such gaps will depend on how many of those gaps happen to obtain in the Humean mosaic. And at least in the case of $\mathcal{S}_{m}$, there's reason to think that the kinds of gaps it posits - namely, appropriately-timed nested measurements - will be quite rare or nonexistent.

It is well-known that setting up a measuring device like $\mathcal{M}_{2}$, which measures some property of the composite $\left[\right.$ atom + vial + cat $\left.+\mathcal{M}_{1}\right]$ system in such a way that we can tell whether the wavefunction has collapsed or not, is extremely difficult. For it to work, the composite system has to remain exceedingly isolated - so much so that it's unclear at present whether this kind of measurement will ever be technologically feasible. Moreover, remember that the failure of $\mathcal{S}_{m}$ to license a prediction about the output of $\mathcal{M}_{2}$ only obtains if the $\mathcal{M}_{2}$ measurement occurs, and is completed within, the window during which it is unclear whether we should say that the $\mathcal{M}_{1}$ measurement has been completed. These appropriately-timed nested measurements are thus not the sort of phenomenon one would expect to occur commonly, or at all, in nature.

If all this is right, then it's fairly likely that there simply won't be any cases of appropriately-timed nested measurements in the Humean mosaic. And so the fact that $\mathcal{S}_{m}$ is unhelpful in licensing predictions about the outcomes of $\mathcal{M}_{2}$ in these cases is not particularly troubling. (Imagine complaining to God that her advice is unhelpful because it doesn't tell you what will happen in situations that never obtain.) From the Humean perspective, then, the fact that $\mathcal{S}_{m}$ has nomological gaps in some exceedingly rare situations should not count as a major strike against it. ${ }^{25}$

\subsection{Strangeness and Explanation}

In sum, none of the ways of explicating the measurement problem gain traction from a Humean perspective. Appeals to "measurement" in the fundamental laws are not a dealbreaker for the Humean in the way they are for an antiHumean. Nevertheless, one might still think that there is an unresolved problem

\footnotetext{
${ }^{25}$ Nomological gaps can plausibly be found even in such respectable theories as general relativity and Newtonian mechanics. Consider the wormhole cases popularized by Thorne (1994), or the dome case made famous by Norton (2003). In both scenarios, the dynamics fails to specify probabilities for the various theoretically-possible outcomes. Given the rarity of the conditions generating these scenarios (closed timelike curves, particles perched perfectly atop spherical domes), the Humean would plausibly be fine countenancing these gaps-as indeed are practicing physicists. $\mathcal{S}_{m}$ is thus in good theoretical company. (Thanks to an anonymous referee here.)
} 
here. Isn't it after all quite strange that nature acts one way in measurement contexts and another way in non-measurement contexts?

In all likelihood, part of what motivates this "strangeness" worry is the thought that nature is paying attention to whether or not a system is being measured, and directing the behavior of the system accordingly. That would indeed be strange. But remember, this is not the right way to think about things on the Humean view. Nature isn't "paying attention" to whether or not a system is measured; it's not paying attention to anything. Rather, nature is behaving "blindly," as it were, and in doing so it sometimes behaves one way, sometimes another. It just turns out that the most convenient way to characterize which way it behaves in which contexts may appeal to the notion of measurement. Put this way, it should strike us as not so strange after all if the fundamental laws make reference to measurement.

Nevertheless, it would be unwise to dismiss this worry too quickly. Perhaps what is really sought here is a scientific, mechanical explanation for why nature acts as if it is paying attention to whether or not a measurement is occurring - for why wavefunctions collapse, or appear to collapse, upon measurement. Physicists are certainly inclined to search for such explanations, as the literature on interpretations of quantum mechanics readily attests. Mustn't there be a scientific explanation of the appearance of wavefunction collapse?

In short, no - at least not on the Humean picture. Suppose it turns out that $\mathcal{S}_{m}$ is the best systematization, so that by the Humean's lights, its regularitiesthe Schrödinger equation and the measurement postulate - are the laws. Then demanding a mechanical explanation of collapse upon measurement is equivalent to demanding an explanation of why the laws are the way they are. It would thus be akin to asking why mass bends spacetime, or why opposite charges attract. Presumably, if there is an explanation for why the laws are the way they are, it will involve appealing to laws that are more fundamental, for it is hard to see how one could explain a necessary law by appealing to contingent conditions.

Two points deserve mention here. First, it is by no means obvious from scientific practice that every law must be explained in terms of some more fundamental law. Indeed, the search for a "theory of everything" indicates that there is an end goal: one unified law from which all the particular phenomena of nature can be derived. Supposing we discovered such a law, it would be perverse to require that even that law must be explained in terms of a more fundamental law, and so on ad infinitum. So scientific practice does not seem to underwrite the demand that any law be explained in terms of more fundamental laws.

Second, and more generally, this demand for further explanation of the laws is not well motivated given the Humean's basic metaphysical picture. The Humean thinks that, fundamentally speaking, all there is to the world is "a vast mosaic of local matters of particular fact, just one little thing and then another" (Lewis, 1986, p. ix). On this picture, laws, causes, dispositions, and counterfactuals are all metaphysically derivative notions whose truth conditions are functions of the content of the Humean mosaic. As Hall (ms, p. 40) points out, it is thus natural to view these notions pragmatically: truths about laws, 
causes, etc. are marked as distinctively appropriate targets for scientific inquiry not because they have some privileged metaphysical status, but rather because they are somehow useful to us in our attempts to navigate the universe.

Notice what this implies about explanation on the Humean picture. While there is no widely accepted account of scientific explanation, there are a variety of proposals. Some explicate it in terms of laws (e.g. Hempel and Oppenheim [1948], Railton [1978], Maudlin [2007], and Lange [2009]), others in terms of causation (e.g. Lewis [1986], Woodward [2003], and Craver [2007]), and still others in terms of counterfactuals (e.g. Reutlinger [2016]). If any of these accounts is on the right track, then on the Humean picture, the pragmatism that infects the notions of laws, causation, and counterfactuals is also going to infect the notion of explanation. In other words, the Humean will also view explanation pragmatically: we search for explanations because they are somehow useful in our attempts to navigate the world. ${ }^{26}$

On this view of scientific explanation, it is by no means obvious that there should be some explanation for why wavefunctions (appear to) collapse in measurement contexts. Whether or not such an explanation exists would seem to depend on whether there is a more useful way of distinguishing the contexts in which the Schrödinger equation and the measurement postulate apply other than appealing to the notion of measurement. In other words, on the Humean picture, the question of whether there is an explanation for nature's disparate behaviors in these different contexts essentially just amounts to the question of whether there is another systematization that (a) does not appeal to measurements, and (b) is better-more useful in our attempts to navigate the universe-than $\mathcal{S}_{m}$.

If there is a better systematization, then we can use it to explain why $\mathcal{S}_{m}$ works as well as it does, and we would thereby have a scientific explanation for why nature behaves differently in measurement and non-measurement contexts. And if there isn't a better systematization, we would likely never be positioned to know that this is the case, so it would still make sense to continue looking for one. This allows the Humean to account for the scientific impulse to search for a scientific explanation of wavefunction collapse: that is just the search for a better systematization than $\mathcal{S}_{m}$. But there might not be a better systematization, and therefore there might not be a scientific explanation of why wavefunctions collapse upon measurement. Scientific explanations come to an end somewhere, and for the Humean they come to an end with the best system.

\section{$5 \quad$ Proliferation of Imprecision?}

One of the most serious objections to this line of reasoning is that it overgeneralizes. If we can use this sort of argument to justify vagueness in the quantum mechanical laws, then what's to stop us from using it all over the place in fundamental physics? Indeed, we might discern the following recipe: take a candidate

\footnotetext{
${ }^{26}$ Bhogal (forthcoming) and Dorst (2019b) both elaborate Humean approaches to scientific explanation along these lines.
} 
fully-precise law from physical practice, and construct a heuristic that conveys the approximate content of that initial precise law in a manner that is much easier to use. This greater ease-of-use is paid for, let us suppose, by various sorts of vagueness in the statement of the heuristic, e.g. in its application conditions, predictions, etc. Where before we had exact equalities, now we have approximate equalities. In this case doesn't the argument I have just been rehearsing give us reason to think that the real fundamental law will be the vague heuristic rather than the fully precise principle that we started with? And isn't this completely at odds with actual physical practice? ${ }^{27}$

This sort of objection is elaborated by Woodward (2014), developing an argument initially suggested by Roberts (2008, pp. 9-10) against the BSA as a whole. Woodward argues that the kind of balance between simplicity and strength that it recommends is not one that we can find to appreciable degrees in actual scientific practice. He suggests, rather, that the kind of balance we find in scientific practice is closer to a lexical ordering, where strength almost always outweighs simplicity, and the latter figures more prominently in deciding between competing hypotheses of nearly-equal strength. If this is right, then our Humean dissolution of the measurement problem, which gives a more significant role to simplicity and tractability, would appear to rely on a facet of the BSA that was antecedently problematic and stands in need of rectification.

The flat-footed response here would just be to insist that Woodward is wrong: we do find something like the balance recommended by the BSA in scientific practice. After all, physics is willing to countenance chances, and the story the BSA tells about the motivation to posit chance is precisely that of a balance between simplicity and strength. Chances enter the picture, not as fundamental elements of the mosaic, but rather as posits of the best system meant to convey a lot of information very succinctly. (Think, for example, of one systematization that lists all the outcomes of flips of every fair coin, and another that just posits that all such coins have a $50 \%$ chance of landing either way. The traditional Humean story about chance is that the latter can earn its status as a stochastic law by achieving a massive gain in informativeness at an acceptable cost to strength.) The fact that we are willing to posit chances, one might think, shows that we are willing to make significant tradeoffs of strength for simplicity.

But in other contexts, Woodward is clearly right. As a simple case, consider the law of universal gravitation:

$$
F_{g}=G \frac{m_{1} m_{2}}{r^{2}}
$$

where $G$ is the gravitational constant of value $6.67430 \times 10^{-11} \mathrm{~m}^{3} \cdot \mathrm{kg}^{-1} \cdot \mathrm{s}^{-2}$. Suppose that we round the value of $G$ to the nearest tenth to get $G^{\prime}=6.7 \times 10^{-11}$ $\mathrm{m}^{3} \cdot \mathrm{kg}^{-1} \cdot \mathrm{s}^{-2}$, and then treat that as entering into the real fundamental law:

$$
F_{g} \approx G^{\prime} \frac{m_{1} m_{2}}{r^{2}} .
$$

${ }^{27}$ Thanks to an anonymous referee for pressing this objection. 
No physicist in their right mind would recommend this, despite the fact that doing so would make the law slightly more simple and tractable at some cost to its strength.

So on the one hand, we seem to be willing to make significant tradeoffs of strength for simplicity in the case of chance, whereas on the other hand, we do not even entertain the possibility of making such a tradeoff in stating the values of fundamental constants. Whence the difference?

I think the problem is that we have not been sufficiently clear about exactly what notion of strength ought to be at play in the BSA, and hence about what the purpose of balancing simplicity (and tractability) against strength is meant to be in the first place. Once we see things aright here, it will become clearer why we are willing to make the tradeoff in some cases but not others, and why it is plausible that the tradeoff is acceptable in the case of $\mathcal{S}_{m}$. But all of this will take a bit of setting up.

Return, to start, to the quote from Albert (§3) that was meant to spell out the motivations for balancing simplicity against strength. The guiding idea we found there was that laws are efficient summaries of the Humean mosaic. This was gleaned from roughly the first half of the quote, which was framed as a request for as much information about the world as could be conveyed in a limited amount of time. But the second half of the quote suggests an alternative reading: that the laws are things that help us effectively navigate the world. In this connection, note that it is not entirely obvious that an efficient summary of the mosaic would be maximally helpful to creatures embedded within it attempting to navigate it. ${ }^{28}$ Instead, something that may be more helpful for navigational purposes is a set of principles that embedded agents can use to amplify their information about the world - something that allows them to plug in the limited information that they have, and churns out a great deal more information in return. This line of thought suggests that we view the laws, not as efficient summaries of the mosaic, but rather as information amplifiers: principles that embedded agents can use to amplify their knowledge of the mosaic.

Good information amplifiers have two salient features. First, their ratio of outputs to inputs is high, i.e. they give you a lot of informational bang for your informational buck. This suggests a revised understanding of the notion of strength: a system of laws is strong, not insofar as it directly conveys a lot of information about the mosaic, but insofar as it allows us to extract a lot of information about the mosaic given the information that we already have. ${ }^{29}$

\footnotetext{
${ }^{28}$ See e.g. Dorst (2019a) $\S 3$ for an extended discussion of this point.

${ }^{29}$ Many commentators on the BSA have suggested revising its conception of strength in this direction. The earliest such suggestion that I'm aware of is Earman (1984, p. 198): "Lewis suggests that strength be measured by information. But the practice of science speaks not in favor of strength per se but strength in intended applications... [S]trength as measured by the amount of occurrent fact and regularity explained or systematized relative to appropriate initial/boundary conditions seems closer to actual scientific practice than strength as measured by information content per se." Woodward (2014) himself makes a similar suggestion to circumvent some of his worries, as does Hall (ms, 48 ff.) in distinguishing between the best system's Dynamical Hypothesis and Initial Conditions Hypothesis. Finally, something like
} 
The second feature of good informational amplifiers is that they are compact, i.e. they are not excessively unwieldy and complex. In fact, this is basically already built into the first feature: an amplifier that is inordinately complex effectively has a lower ratio of outputs to inputs, since one of the things you have to know in order to use it to amplify your information is the information contained in the amplifier itself. In other words, one should count the information content of the amplifier itself when evaluating its informational demands. We see here a role for simplicity and tractability: they enter into the calculus to keep the information content of the amplifiers themselves from burgeoning too much, and also to help ensure that we can, in fact, extract a good deal of information from them. Complex and intractable amplifiers would have a low output-to-input ratio because we would be unable to extract much from them.

There is a lot more to say about this re-conceived motivation for balancing simplicity, strength, and tractability. ${ }^{30}$ But for our purposes these general remarks will be sufficient. At this point two things need to be done. First, we need to show how this revised amplifier conception of the best system accommodates physicists' willingness to posit chances without also motivating rounding the values of fundamental constants in law statements. Second, we need to show how it makes $\mathcal{S}_{m}$ a plausible candidate for best system without thereby conflicting with actual physical practice by engendering an unwanted proliferation of imprecision in putative fundamental laws.

The first thing to note is that on this amplifier view, simplicity and tractability work directly on the amplifiers, not on the information about the mosaic that we extract from the amplifiers. Their effect is essentially to compress the amplifiers to decrease their informational demands. But that is consistent with hoping that, in principle, all information about the mosaic can be gleaned from the amplifiers coupled with a relatively small input. Certainly it will be harder to find amplifiers that are simpler and more tractable, and yet still return the entire mosaic as output, but there is no decisive impossibility here.

That said, for some kinds of mosaics, the best amplifiers will have to leave out some information by employing chances. In particular, this will be the case for mosaics that appear sufficiently random such that they cannot be effectively compressed. Any amplifier that does better with respect to its informational outputs (i.e. avoids positing chances, and instead gives us exact outcomes) would either (a) require an enormously greater amount of information as input, or (b)

this notion of strength figures as the keystone in a spate of recent developments of the BSA, e.g. Callender's (2017) account of time in a best system framework, Hicks's (2018) Epistemic Role Account, Dorst's (2019a) Best Predictive System Account, and Jaag and Loew's (2020) Cognitive Usefulness Account.

${ }^{30}$ Much of it, I think, has already been said in the references mentioned in fn. 27 . But one thing that still needs to be said concerns the respect in which something like Lewis's $\forall x F x$ counts as intractable, but the fundamental equations of quantum field theory, say, nevertheless count as tractable. For in practice there is quite a lot about the world that cannot be effectively predicted by appealing to QFT. I take this to be one of the most pressing problems facing the Humean views mentioned in the previous footnote, to which the argument of this paper is clearly tied. This problem is explicitly addressed in Blanchard (forthcoming) and Dorst (forthcoming), but I lack the space to get further into these matters here. 
contain a great deal of information content itself. ("Want to know whether this coin will land heads? Just locate it within this Almanac of every coin flip in the entire mosaic to find out!") Thus we can see why, given certain mosaics, it would be reasonable to countenance chances in the best system. For such mosaics, positing chances may involve a decrease in the output capacity of the system, but it achieves a far greater decrease in the input demands, achieving a better overall ratio of outputs to inputs.

On the other hand, rounding off the gravitational constant drastically reduces the output capacity of the law of universal gravitation. That law is meant to apply to every particle at all times. The precise version gives us something like a sharp image of the entire mosaic (at least with respect to the gravitational force). By contrast, to round off the gravitational constant and adopt the approximate version of this law would be to blur out that entire image, such that only its gross features remain discernible. The inclusion of extra digits in the statement of the law is a minuscule price to pay, in terms of the informational content of the amplifier, compared to the increased image quality. This tradeoff, in other words, isn't even worth considering.

Let's recap where we've come to. In the cases of the heuristics that we imagined above, we started with a fully precise candidate law and moved to an imprecise heuristic that was simpler and more tractable. In doing so, however, we lost a great deal of information about the mosaic - we went from a law that provided a sharp image of the mosaic to one that provided a blurry image. And we noted that physicists rarely if ever countenance this kind of tradeoff. To accommodate this within the BSA, we had to change our understanding of the motivation for balancing simplicity, strength, and tractability: the goal is not to find an efficient summary of the mosaic, but rather to find good amplifiers: principles that take as input some information about the mosaic and produce as output a great deal more information about the mosaic. The best possible amplifying system will be one that requires minimal informational input (preferably of the types that we are likely to have access to) and allows us to extract from it the greatest amount of informational output. ${ }^{31}$

Now let's return to $\mathcal{S}_{m}$. I want to maintain that $\mathcal{S}_{m}$ is a plausible candidate for best system. How does this revised amplifier conception of the BSA allow this without also motivating the thought that any heuristic may be a fundamental law?

The key point is to note that there is a significant disanalogy between the heuristic cases we imagined above and the case of $\mathcal{S}_{m}$. In the heuristic cases, like

\footnotetext{
${ }^{31}$ One way to complicate this view would be to introduce distinctions in the kinds of information that we care most about, and then maintain that the outputs of a better amplifier system have a higher proportion of this "premium" information. One might think, for instance, that other things being equal we would prefer to have more information about the future than about the past, or that we would prefer to have more information about happenings on Earth than about happenings on some undiscovered planet in an unobservable corner of the universe. I won't pursue this suggestion further here. Implementing it would make the resulting account of laws even more aggressively pragmatic, though Albert's quote shows that elements of pragmatism were already latent in the guiding idea of the BSA from the very beginning.
} 
that of our approximate version of the law of universal gravitation (4), we had available an alternative principle (3) that was more precise. Physicists would never choose (4) when they have (3) available instead. Why? According to the amplifier conception of the BSA, the answer is that the amount of information about the mosaic that we can extract from (3) is far greater than the amount we can extract from (4), whereas the increased informational demands of (3) are negligible.

If $\mathcal{S}_{m}$ is analogous to (4), is there a system analogous to (3) that is more precise and more informative about the mosaic? One suggestion would be $\mathcal{S}_{c e}$. It is true, of course, that $\mathcal{S}_{c e}$ 's informativeness about the mosaic is significantly greater than that of $\mathcal{S}_{m}$. If we stuck with Lewis's original conception of strength (i.e. how much the system directly tells us about the mosaic) and accepted Woodward's argument that strength is lexically prior to simplicity, then it would be puzzling why $\mathcal{S}_{c e}$ is not in the running for best system. But on the amplifier conception, there is no puzzle at all: $\mathcal{S}_{c e}$ scores very poorly as an amplifying system because of the amount of information built into it - its informational demands are far higher than those of $\mathcal{S}_{m}$. Opting for $\mathcal{S}_{c e}$ over $\mathcal{S}_{m}$ would be akin to choosing the coin flip almanac as opposed to positing chances.

Setting aside $\mathcal{S}_{c e}$, we have no principle analogous to (3) in this case. That is, we have no reasonably effective amplifying system from which we can extract appreciably more information about the mosaic than we can from $\mathcal{S}_{m}$. What we do have in alternative interpretations like GRW and Many Worlds and Bohmian Mechanics are candidate theories where the amplifying systems themselves are stated in more precise terms, without appeal to vague notions like "measurement." But in these cases, greater precision in the statement of the systematizations themselves doesn't allow us to extract a greater amount of information about the mosaic. Rather the alternative interpretations are, for all practical purposes (FAPP), empirically equivalent to $\mathcal{S}_{m} \cdot{ }^{32}$ In effect, this is just the familiar point that it is difficult to settle the question of interpretation empirically. As Albert helpfully sums up, "[D]ifferent conjectures about precisely where and precisely when collapses occur are the sorts of conjectures which (for all practical purposes; or, rather, for all presently practical purposes) cannot be empirically distinguished from one another" (1992, p. 91).

In short, what we have with $\mathcal{S}_{m}$ is a highly unusual case, one where imprecision in the statement of the laws isn't hurting its informativeness about the mosaic relative to the other more precise candidate systems that are comparably undemanding in terms of informational input. This is disanalogous to the heuristic cases we were imagining above; there, the imprecision in a principle like (4) significantly hampers its informativeness compared to a principle like (3), whose informational demands are not radically greater than those of (4).

At the risk of belaboring the point, let me make one more analogy that may prove helpful. Suppose the mosaic is comprised of a bunch of coin flips,

\footnotetext{
${ }^{32}$ Of course, they are not exactly empirically equivalent: there are possible cases like those of our nested measurements from $\S 4.2$ where their predictions would diverge from $\mathcal{S}_{m}$. But as I've already argued, such cases are so rare that they should not weight heavily against $\mathcal{S}_{m}$ in terms of its amplifying power.
} 
with roughly equal frequencies of heads and tails. How best to systematize it? Consider three options: (1) a system that directly reports the outcome of every coin flip; (2) a chancy system that posits that each coin has a $50 \%$ chance of landing either way; (3) a deterministic system that says that the outcome of each flip depends on minute differences in boundary conditions right before each toss, but gives us good reason to think that those boundary conditions will be distributed such that coins comes up heads roughly $50 \%$ of the time.

Given what I've said about the mosaic, we would probably take (2) and (3) seriously, but certainly not (1). The amplifier conception makes sense of this. (1) is a terrible amplifier because of the amount of information contained within it. By contrast, (2) and (3) are comparably good amplifiers - at least assuming that we are typically not going to be in position to examine the initial conditions of each flip extremely precisely. If that assumption is right, then (2) and (3) allow us to extract a similar amount of information about the mosaic. Of course, (3) implies that if we could position ourselves better with respect to the initial conditions of each toss, then we could extract more information about the outcomes; but in practice (let's suppose) we can do no better with (3) than we can with (2). In that situation we would take them roughly equally seriously.

We have an exactly analogous situation here, with $\mathcal{S}_{c e}$ corresponding to (1), $\mathcal{S}_{m}$ corresponding to (2), and GRW, Many Worlds, and Bohmian Mechanics corresponding to (3). The informational demands of $\mathcal{S}_{c e}$ rule it out as a good amplifier. And while GRW et. al. are more precise than $\mathcal{S}_{m}$, in practice the amount of information that we can extract from them is the same. The amplifier conception thus recommends that we take them roughly equally seriously.

This point also helps to address a related worry, which is that "being measured" is a very derivative property-much moreso than the examples of acceleration and resultant force that Hicks and Schaffer use to motivate property opportunism. Is there a limit on how derivative a property may be and still figure into fundamental laws? The answer suggested here is that in principle there is no limit. It will always depend on the nature of the mosaic, the other systematizations available, and whether the inclusion of the derivative property in the candidate fundamental laws engenders an appreciable loss in ampliative power relative to those other systematizations. The bar is low here; in most cases, the loss in strength will be significant enough to rule out that candidate system. But in certain unusual cases, like the present one, it might not be.

However, it might be thought that the alternative interpretations are more informative about the mosaic because they tell us more about the fundamental ontology. While the ontology of the GRW theory is debated, that of Many Worlds and Bohm's theory is clear: Many Worlds posits a universal wavefunction, and Bohmian Mechanics posits particles and a wavefunction moving them around. Even if the empirical predictions we can extract from these candidate systems aren't appreciably different from the empirical predictions we can extract from $\mathcal{S}_{m}$, nevertheless the clearer ontological picture that results from these interpretations tells us more about the kinds of things that are going on in the mosaic than we get from $\mathcal{S}_{m}$. 
The problem with this objection is that it elides the distinction between (1) an interpretation of quantum mechanics and (2) the best systematization of the mosaic. The best system just reports the laws; an interpretation of quantum mechanics proposes both laws and a fundamental quantum ontology. It is not the business of the laws themselves to describe the fundamental ontology.

Take, for example, Newton's laws of motion. These are formulated quite generally, without referencing any fundamental kinds of entities. They refer to things like forces, masses, relative distances, and accelerations, but these can be properties of any physical system, not just the fundamental ones. Richard Feynman (1965) has likewise emphasized that most laws are, in a sense, subject neutral: they do not specify the sorts of entities that they apply to. Throughout his wonderful lectures in The Character of Physical Law, he discusses various laws of nature, before opening the last lecture with the following remark:

You may think I have told you everything already, because in the lectures I have told you all the great principles that are known. But the principles must be principles about something; the principle of the conservation of energy relates the energy of something, and the quantum mechanical laws are quantum mechanical laws about something - and all these principles added together still do not tell us what the content is of the nature that we are talking about. I will tell you a little, then, about the stuff on which all of these principles are supposed to have been working. (1965, p. 149, italics in original)

Though GRW, Many Worlds, and Bohmian Mechanics all tell us more about the fundamental ontology than does $\mathcal{S}_{m}$, the laws of those theories, strictly speaking, do not. ${ }^{33}$ It is not legitimate to compare the informativeness of a full-fledged interpretation of quantum mechanics, replete with laws and a fundamental ontology, with the informativeness of $\mathcal{S}_{m}$-a candidate best system of laws - by itself.

Still, this raises the question of how a Humean sympathetic with this dissolution of the measurement problem would approach the question of interpretation. We turn to this question in the next section.

\section{Humean Interpretation of Quantum Mechanics}

Intuitively speaking, an interpretation of quantum mechanics is meant to provide a picture of what the world is like if quantum mechanics is true of it. More precisely, it should describe the fundamental ontology and the laws governing it. In doing so, part of the goal is typically to solve the measurement problem

\footnotetext{
${ }^{33}$ This is another respect in which the amplifier conception of the best system is in better agreement with physical practice than the efficient summary conception. On the latter conception, it would seem plausible that the best system, in compactly conveying a lot of information about the mosaic, should tell us about the fundamental ontology. By contrast, this is less plausible on the amplifier conception, where the best system may take initial or boundary conditions of that ontology as input, and produce more such conditions as output. But to accomplish this it needn't tell us, by itself, what the fundamental ontology is.
} 
by showing how the laws engage with the fundamental ontology to bring about wavefunction collapses, or apparent collapses (as the case may be).

Traditionally, then, the dynamics and ontology are quite closely connected: the dynamics must be formulated in the language of the fundamental ontology, so the laws cannot directly appeal to "measurements." This does not imply that the laws themselves must tell us directly what the fundamental entities are (consistent with Feynman's remarks in the previous section). But it does imply that if we want to understand why collapses occur, we must have a clear view of the fundamental ontology, and the properties it can instantiate, so that we can understand how the laws will engage with those properties and precipitate (apparent) collapses. A successful interpretation of quantum mechanics provides us with a mechanistic explanation, in terms of the fundamental ontology obeying the fundamental dynamics, of (the appearance of) wavefunction collapse. Solving the measurement problem is thus inextricably tied to identifying the ontology and properties of the quantum world.

If my arguments above are successful, the Humean need not approach the project of interpreting quantum mechanics in exactly this way. In particular, since the laws can appeal to non-fundamental properties, they are allowed to reference "measurements" in their characterization of when wavefunction collapse occurs. Thus we no longer require a specification of the conditions of wavefunction collapse in the language of the fundamental ontology, and we can seriously countenance candidate statements of the laws that resolve the measurement problem (if it can even be called a "problem" here) without having a clear picture of the underlying ontology. ${ }^{34} 35$

None of this is to say that Humeans can forgo altogether the project of providing an interpretation of quantum mechanics. An interpretation is still necessary to provide the ontology and thereby specify the contents of the mosaic itself. However, this point gives rise to a natural worry: how can the Humean possibly resolve the measurement problem without having a clear picture of the mosaic? After all, if the laws are determined by the best systematization of the mosaic, don't we need to know what the mosaic is before we can know whether an appeal to the notion of "measurement" in that systematization is worth it?

In this regard, let me reiterate that my goal is not to argue that we can know that $\mathcal{S}_{m}$ is the best system, but to argue that it may be, and in particular to argue that it is not ruled out simply because it includes the measurement

\footnotetext{
${ }^{34}$ If this strikes you as scientifically unrealistic, note that it's exactly what has happened in the case of quantum mechanics itself: we've long had a proposal for the quantum mechanical laws (i.e. the Schrödinger equation and the measurement postulate) without having a clear view of the underlying ontology.

${ }^{35}$ Even the fundamental space of the world need not be settled. Because the wavefunction is defined on configuration space, some authors have argued that we must take the world's fundamental space to be structurally isomorphic to configuration space-see, e.g., Albert (1996) and North (2013). Others have been more cautious; Maudlin (2013), for example, argues that given the wavefunction's role in Bohmian mechanics, it is naturally represented by a scalar function on configuration space, regardless of what kind of physical thing it is. My point here is not to take a stand on this issue. I only mean to emphasize how much may be undecided about the nature of the mosaic even after the Humean has a definite candidate system of laws in hand.
} 
postulate. Of course, it might be ruled out for other reasons - e.g. it may end up being inconsistent with things that happen in future experiments. The question then is not, "Can we conclusively show that $\mathcal{S}_{m}$ is the best system without knowing the full contents of the mosaic?" but rather, "Given what we do know about the mosaic, is $\mathcal{S}_{m}$ a plausible candidate for best system?"

I think the answer is yes. Even though we don't have knowledge of the fundamental contents of the mosaic, we do have knowledge that constrains what those contents are like. Specifically, we know that whatever the fundamental entities are and however they behave, they arrange themselves in such a way as to produce all the results of our quantum experiments. We know, then, that whatever the fundamentalia are, they arrange themselves into systems that appear to behave in radically different ways depending on whether they are being measured or not. That is enough to make $\mathcal{S}_{m}$ a plausible candidate for best system, though of course it does not prove that it is the best.

Again, ultimately the Humean cannot avoid providing an interpretation of quantum mechanics, since the specification of the mosaic depends upon it. However, if my arguments in this paper are right, an acceptable Humean interpretation of quantum mechanics might still appeal to the notion of measurement in its statement of the laws. Of course, the dominant interpretations nowadays expressly avoid doing so. Objective collapse theories like GRW, for example, appeal to a probabilistic collapse process that is overwhelmingly likely to occur whenever a measurement is made, but the concept of measurement plays no crucial role in spelling out exactly when these collapses occur. On the other hand, Bohmian Mechanics and Many Worlds posit no collapse process, and instead rely on the notion of decoherence to articulate when an effective collapse occurs. Since these processes tend to occur in measurement contexts, no explicit appeal to the notion of measurement is required in these cases either.

This assiduous avoidance of appeals to measurement is due to the fact that proponents of these theories have traditionally regarded such appeals as illegitimate in the laws of a fundamental physical theory. But if, as I have argued, such appeals are actually fair game for the Humean, then there are further interpretive possibilities than we were previously countenancing. For instance, if the mechanistic explanation of (genuine or apparent) wavefunction collapse given by any of these interpretations turns out not to be tenable, it is not out of the question that a Humean would substitute something like the measurement postulate in its place, while maintaining the same basic ontology. One can imagine, for example, a GRW-like theory where the probabilistic flashes are replaced by measurement-induced collapses, or a relative of Many Worlds where the branching events are precipitated by measurements. Admittedly, there would be plenty of issues to be resolved on these views. My point is not that they would be entirely unproblematic, nor that integrating an overt appeal to "measurement" in the laws of these theories would be straightforward, nor indeed that the resultant theories would have all the same theoretical virtues as their measurement-free counterparts. It is rather that, for the Humean, and only for the Humean, there is no in-principle prohibition against using a vague term like "measurement" in the characterization of the laws. It may, and arguably 
does, earn its keep.

\section{Conclusion}

Authors concerned with the relationship between quantum mechanics and the Humean account of laws have often focused on the question of whether the lessons of quantum mechanics are compatible with Lewis's characterization of the Humean mosaic. Are the fundamental properties really local, intrinsic, and nonmodal, as Lewis maintained? Quantum mechanics has given us at least some reason to doubt this. ${ }^{36}$

While these worries certainly deserve the Humean's attention, my aim here has been to point out that there is another aspect of quantum mechanics that squares quite well with Humeanism, namely the explicit appeal to "measurement" in its laws. Indeed, if we take traditional quantum mechanics at face value, the fact that it makes such appeals could be regarded as strong support for Humeanism over anti-Humeanism. For the Humean can allow that the notion of "measurement" figures into the fundamental laws of nature, but the anti-Humean cannot: if anti-Humeanism is right, then textbook quantum mechanics is simply a non-starter - it has no chance of giving us the fundamental laws. ${ }^{37}$

Perhaps, then, the conflict between quantum mechanics and Humeanism has been overstated. Whereas some aspects of quantum mechanics are troubling for the Humean - especially for the Humean committed to a Lewisian picture of the mosaic - other aspects have turned out to be uniquely concordant with a Humean metaphysics.

\footnotetext{
${ }^{36}$ Loewer (1996) and Maudlin (2007) articulate some of the main worries here. Miller (2014) and Bhogal and Perry (2017) provide some illuminating responses to these worries.

${ }^{37}$ Though see Chen's (forthcoming a) discussion of "minimal non-Humeanism" for a potentially dissenting view.
} 


\section{References}

[1] Albert, D. (1992). Quantum Mechanics and Experience. Cambridge: Harvard University Press.

[2] Albert, D. (1996). "Elementary quantum metaphysics." In J. T. Cushing, A. Fine, and S. Goldstein (Eds.), Bohmian Mechanics and Quantum Theory: An Appraisal (pp. 277-284). Dordrecht: Kluwer.

[3] Albert, D. (2015). After Physics. Cambridge: Harvard University Press.

[4] Allahverdyan, A. E., Balian, R., \& Nieuwenhuizen, T. M. (2013). "Understanding quantum measurement from the solution of dynamical model." Physics Reports, 525, 1-166.

[5] Allahverdyan, A. E., Balian, R., \& Nieuwenhuizen, T. M. (2017). "A sub-ensemble theory of ideal quantum measurement processes." Annals of Physics, 376, 324-352.

[6] Armstrong, D. (1978). Universals and Scientific Realism, Volumes 1 and 2. Cambridge: Cambridge University Press.

[7] Armstrong, D. (1983). What is a Law of Nature? Cambridge: Cambridge University Press.

[8] Beebee, H. (2000). "The non-governing conception of laws of nature." Philosophy and Phenomenological Research, 61, 571-594.

[9] Bell, J. S. (1990). "Against 'measurement."' Physics World, 3, 33-40.

[10] Bhogal, H. \& Perry, Z. (2017). "What the Humean should say about entanglement." Noûs, 51, 74-94.

[11] Bhogal, H. (2021). "Nomothetic explanation and Humeanism about laws of nature." In K. Bennett \& D. Zimmerman (Eds.), Oxford Studies in Metaphysics, Volume 12 (pp. 164-202). New York: Oxford University Press.

[12] Blanchard, T. (forthcoming). "Best system laws, explanation, and unification." In M. Hicks, S. Jaag, \& C. Loew, (Eds.), Humean Laws for Human Agents. Oxford: Oxford University Press.

[13] Bohr, N., \& Kalckar, J. (1985). Niels Bohr: Collected Works, Volume 6: Foundations of Quantum Physics 1 (1926 - 1932). Amsterdam: Elsevier.

[14] Callender, C. (2017). What Makes Time Special? Oxford: Oxford University Press.

[15] Carpenter, R. H. S. \& Anderson, A. (2006). "The death of Schrödinger's cat and of consciousness-based quantum wave-function collapse." Annales de la Fondation Louis de Broglie, 31, 45-52. 
[16] Carroll, S. (forthcoming). "Reality as a vector in Hilbert space." In V. Allori (Ed.), Quantum Mechanics and Fundamentality: Naturalizing Quantum Theory Between Scientific Realism and Ontological Indeterminacy. Synthese Library: Springer.

[17] Cartwright, N. (1999). The Dappled World: A Study of the Boundaries of Science. Cambridge: Cambridge University Press.

[18] Chen, E. K. (forthcoming a). "The past hypothesis and the nature of physical laws." In B. Loewer, E. Winsberg, \& B. Weslake (Eds.), Time's Arrow and the Probabillity Structure of the World. Cambridge: Harvard University Press.

[19] Chen, E. K. (forthcoming b). "Fundamental nomic vagueness." The Philosophical Review.

[20] Cohen, J. \& Callender, C. (2009). "A better best system account of lawhood." Philosophical Studies, 145, 1-34.

[21] Craver, C. (2007). Explaining the Brain: Mechanisms and the Mosaic Unity of Neuroscience. New York: Oxford University Press.

[22] Deumens, E. (2019). "On classical systems and measurements in quantum mechanics." Quantum Studies: Mathematics and Foundation, 6, 481-517.

[23] Dirac, P. A. M. (1948). Quantum Mechanics: 3rd Edition. Oxford: Oxford University Press.

[24] Dorst, C. (2019a). "Towards a best predictive system account of laws of nature." British Journal for the Philosophy of Science, 70, 877-900.

[25] Dorst, C. (2019b). "Humean laws, explanatory circularity, and the aim of scientific explanation." Philosophical Studies, 176, 2657-2679.

[26] Dorst, C. (forthcoming). "Predictive infelicities and the instability of predictive optimality." In M. Hicks, S. Jaag, \& C. Loew, (Eds.), Humean Laws for Human Agents. Oxford: Oxford University Press.

[27] Esfeld, M. (2020). "Super-Humeanism: the Canberra plan for physics." In D. Glick, G. Darby, \& A. Marmodoro (Eds.), The Foundation of Reality: Fundamentality, Space, and Time. New York: Oxford University Press.

[28] Feynman, R. (1965). The Character of Physical Law. New York: Modern Library.

[29] Hall, N. (manuscript). "Humean reductionism about laws of nature." Accessed at: https://philpapers.org/archive/HALHRA.pdf

[30] Hempel, C. \& Oppenheim, P. (1948). "Studies in the logic of explanation." Philosophy of Science, 15, 135-175. 
[31] Hicks, M. \& Schaffer, J. (2017). "Derivative properties in fundamental laws." British Journal for the Philosophy of Science, 68, 411-450.

[32] Hicks, M. (2018). "Dynamic Humeanism." British Journal for the Philosophy of Science, 69, 987-1007.

[33] Hughes, R. I. G. (1989). The Structure and Interpretation of Quantum Mechanics. Cambridge: Harvard University Press.

[34] Ismael, Jenann. (2015). "Quantum mechanics." In E. N. Zalta (Ed.), The Stanford Encyclopedia of Philosophy. Accessed at: https://plato.stanford.edu/archives/spr2015/entries/qm/.

[35] Jaag, S. \& Loew, C. (2020). "Making best systems best for us." Synthese, $197,2525-2550$.

[36] Landau, L. D. \& Lifshitz, E. M. (1977). Quantum Mechanics: NonRelativistic Theory. New York: Pergamon Press.

[37] Lange, M. (2009). Laws and Lawmakers. New York: Oxford University Press.

[38] Lewis, D. (1973). Counterfactuals. Malden: Blackwell.

[39] Lewis, D. (1986). Philosophical Papers, Volume II. New York: Oxford University Press.

[40] Lewis, D. (1994). "Humean supervenience debugged." Mind, 103, 474-90.

[41] Lewis, P. (1995). "GRW and the tails problem." Topoi, 14, 23-33.

[42] Lewis, P. (2016). Quantum Ontology. New York: Oxford University Press.

[43] Loewer, B. (1996). "Humean supervenience." Philosophical Topics, 24, 101127.

[44] Loewer, B. (2007). "Laws and natural properties." Philosophical Topics, $35,313-328$.

[45] Loewer, B. (2020). "The package deal account of laws and properties." Synthese. doi: 10.1007/s11229-020-02765-2

[46] Maudlin, T. (1995). "Three measurement problems." Topoi, 14, 7-15.

[47] Maudlin, T. (2007). The Metaphysics within Physics. New York: Oxford University Press.

[48] Maudlin, T. (2013). "The nature of the quantum state." In A. Ney \& D. Albert (Eds.), The Wave Function: Essays on the Metaphysics of Quantum Mechanics (pp. 126-153). New York: Oxford University Press. 
[49] Maudlin, T. (2019). Philosophy of Physics: Quantum Theory. Princeton: Princeton University Press.

[50] Miller, E. (2014). "Quantum entanglement, Bohmian mechanics, and Humean supervenience." Australasian Journal of Philosophy, 92, 567-583.

[51] North, J. (2013). "The structure of a quantum world." In A. Ney \& D. Albert (Eds.), The Wave Function: Essays on the Metaphysics of Quantum Mechanics (pp. 184-202). New York: Oxford University Press.

[52] Norton, J. (2003). "Causation as folk science." Philosopher's Imprint, 3, $1-22$.

[53] Railton, P. (1978). "A deductive-nomological model of probabilistic explanation." Philosophy of Science, 45, 206-226.

[54] Reutlinger, A. (2016). "Is there a monist theory of causal and non-causal explanations? The counterfactual theory of scientific explanation." Philosophy of Science, 83, 733-745.

[55] Roberts, J. (2008). The Law-Governed Universe. New York: Oxford University Press.

[56] Thorne, K. (1994). Black Holes and Time Warps: Einstein's Outrageous Legacy. New York: W. W. Norton.

[57] Vaidman, L. (2020). "Derivations of the Born rule." In M. Hemmo \& O. Shenker (Eds.), Quantum, Probability, Logic (pp. 567-584). Jerusalem Studies in Philosophy and History of Science.

[58] Woodward, J. (2003). Making Things Happen. New York: Oxford University Press.

[59] Woodward, J. (2014). "Simplicity in the best systems account of laws of nature." British Journal for the Philosophy of Science, 65, 91-123.

[60] Wigner, Eugene. (1963). "The problem of measurement." American Journal of Physics, 31, 6-15. 